\title{
Medial Circumflex Femoral Vein
}

National Cancer Institute

\section{Source}

National Cancer Institute. Medial Circumflex Femoral Vein. NCI Thesaurus. Code C52688.

A circumflex femoral vein that accompanies the medial circumflex femoral artery. 